\title{
Nível mínimo de discriminação de componente para caracterização de misturas bifásicas (petróleo/água salgada ou gás) usando transmissão de raios-x ou gama
}

\section{Minimum level of component discrimination for characterization of biphasic mixtures (oil / brine or gas) using gamma and $\mathrm{x}-$ ray transmission}

\author{
Luiz Diego Marestoni ${ }^{1}$; Carlos Roberto Appoloni²; Jair Romeu Eichlt ${ }^{3}$
}

\section{Resumo}

Neste trabalho propomos o conceito de Nível Mínimo Discriminação de Componentes (NMDC) para determinar a discriminação de componentes de uma mistura bifásica. A modelagem matemática seguida da comprovação experimental ilustra o conceito de diferentes misturas utilizando medições de transmissão de cinco linhas de uma fonte ${ }^{241} \mathrm{Am}$. Com base nas simulações, foram elaboradas tabelas de discriminações mínimas. Essas tabelas de discriminação são as previsões teóricas ideais para a determinação experimental, uma vez que fornecem a percentagem mínima de discriminação para cada linha de energia. A simulação foi testada usando uma caixa com paredes de epóxi reforçado com fibra de carbono, para as linhas de energia de 20,8 e $59,54 \mathrm{keV}$.

Palavras-chave: transmissão de raios gama, petróleo e misturas bifásicas

\begin{abstract}
In this paper we propose the concept of Minimum Level of Component Discrimination (NMDC) to determine the components discrimination in a biphasic mixture. A mathematical modeling followed by experimental corroboration illustrates the concept for different mixtures using transmission measurements of five lines from a ${ }^{241} \mathrm{Am}$ source. Based on the simulations, minimum discrimination tables were done. These discrimination tables are the ideal theoretical predictions to lead experimental measurement, since they supply the minimum discriminative percentage for each energy line. The simulation was tested using the box with epoxy walls reinforced with carbon fiber, for the 20.8 and $59.54 \mathrm{keV}$ energy lines.
\end{abstract}

Key words: gamma ray transmission, oil and biphasic mixtures.

\footnotetext{
1 Guaduado e mestre em Física, Universidade Estadual de Londrina; Aluno de Doutorado em Química da Universidade Estadual Paulista; Docente do Departamento de Química e Meio Ambiente do Instituto Federal do Mato Grosso; luiz.marestoni@blv.ifmt. edu.br

2 Docente do Departamento de Física da Universidade Estadual de Londrina - DEFIS-UEL; appoloni@uel.br

3 Mestre em Física, Universidade Estadual de Londrina
} 


\section{Introdução}

O Brasil possui $6,43 \times 10^{12} \mathrm{~m}^{2}$ de bacias sedimentares, com $4,83 \times 10^{12} \mathrm{~m}^{2}$ na terra e $1,55 \times 10^{12}$ $\mathrm{m}^{2}$ na plataforma continental. A principal fonte para a formação do petróleo foi o fitoplâncton do período do Jurássico Superior-Cretáceo, que, quando morreu, foi depositado no fundo dos oceanos e tornou-se parte dos sedimentos e formaram os folhelhos ricos em matéria orgânica.

Para a formação do petróleo é necessário que as bacias tenham sido formadas em condições muito específicas. Por exemplo, rochas sujeitas a temperaturas de $323 \mathrm{~K}$ de 30 mil anos não seriam suficientemente adequado para gerar petróleo, mas apenas o metano biogênico. Em igual período, rochas aquecidas até $463 \mathrm{~K}$ podem gerar gás natural.

Variações no gradiente geotérmico, na profundidade e na duração do aterramento podem gerar variações na bacia sedimentar (STRAPASSON, 2010) e diferentes composições de petróleo. Os processos de extração de petróleo estão evoluindo e ficando cada vez mais acelerados, levando a necessidade de equipamentos mais modernos e eficazes e processos para todas as áreas, desde a extração até o produto final e, além disso, o custo deve ser levado em consideração.

É comum, no processo de extração do petróleo no mar (offshore), a extração de água salgada e/ ou gás natural junto com o petróleo. A indústria do petróleo requer um medidor de fluxo multifásico para determinar as taxas de fluxo de sal, de petróleo e gás em dutos de petróleo (ROACH et al., 1994). O trabalho foi focado neste tipo de mistura, pois a maioria das estações de petróleo no Brasil está no offshore.

A solução tradicional para determinar a quantidade de cada componente da mistura é a de separar os componentes e, em seguida, medir a quantidade de cada um usando uma única fase instrumental (JOHANSEN; JACKSON, 2000). No entanto, este processo é lento, caro, volumoso e solicita que as três fases (petróleo, gás e água salgada) estejam devidamente separadas. Uma possível solução para este problema é a medição em linha da mistura, através do processo de gama ou densitometria de raios- $\mathrm{X}$.

Uma vez que existem estudos na literatura para determinar as substâncias em uma mistura com o uso de uma fonte radioativa, a maior parte dos trabalhos usando a fonte ${ }^{241}$ Am (PUTZ et al., 1993; ROACH et al., 1994; SCHEERS; SLIJKERMAN, 1996; TJUGUM; JOHANSEN; HOSTAD, 2001), neste trabalho o objetivo foi desenvolver um modelo matemático para determinar qual a linha de energia da fonte ${ }^{241} \mathrm{Am}$ que melhor discrimina uma substância dentro de uma mistura e qual o nível mínimo de discriminação para a mistura petróleo / água salgada / gás, bem como a realização de medições de testar as equações desenvolvidas.

\section{Metodologia}

A densitometria de raios gama e de raios $\mathrm{X}$ considera a atenuação do $\mathrm{X}$ e raios gama que atravessa a amostra (I/Io). Para a determinação da fração de uma substância em uma mistura bifásica um único feixe é suficiente. A figura 1 representa o diagrama de modelagem matemática, utilizando uma mistura bifásica de duas substâncias hipotéticas s e m.

Figura 1. Modelo esquemático usado para a modelagem matemática.

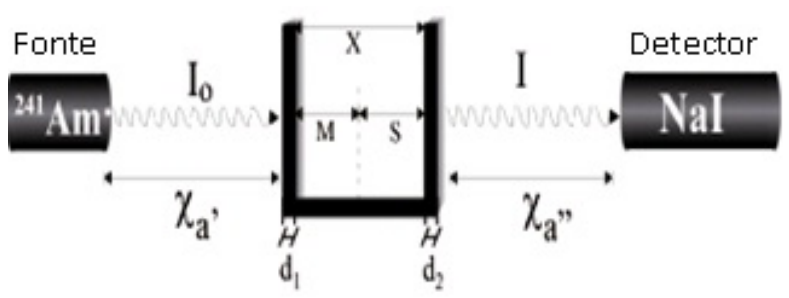

Onde $\mathrm{M}$ representa a espessura da substância $m$ no interior da amostra, $\chi_{a}$, e $\chi_{a}$, representa a espessura das camadas de ar entre a fonte/amostra e amostra/fonte, respectivamente, $\mathrm{X}$ é a espessura total da amostra, que neste trabalho é fixado em $\mathrm{X}$ 
$=25 \mathrm{~mm}, \mathrm{~S}$ é a espessura da substância s que está dentro da amostra, $\mathrm{d}_{1}+\mathrm{d}_{2}=\mathrm{d}$ representa a espessura total da caixa (acrílica ou epóxi), que neste trabalho foi fixada em $\mathrm{d}=4,0 \mathrm{~mm}$, Io e I são as intensidade do feixe incidentes e transmitido pela amostra, respectivamente.

De acordo com o mostrado na figura 1, a partir da fração feixe que foi atenuada (I/Io), das densidades dos materiais envolvidos $(\rho)$ e dos coeficientes de atenuação $(\mu)$, a fração de uma substância $S$ pode ser determinada pela equação:

$$
(\mathrm{I} / \mathrm{Io})=\exp \left[-\mu_{\mathrm{cx}} \rho_{\mathrm{cx}} \mathrm{d}-\mu_{\mathrm{s}} \rho_{\mathrm{s}} \mathrm{S}-\mu_{\mathrm{a}} \rho_{\mathrm{a}}\left(\chi_{\mathrm{a}}, \chi_{\mathrm{a}},\right)-\mu_{\mathrm{m}} \rho_{\mathrm{m}}(\mathrm{S}-\mathrm{X})\right]
$$

O programa WinXcom(GERWARD et al., 2001) foi usado para calcular as seções de choque e os coeficientes de atenuação de massa das combinações e misturas desejadas. Para as medições foi utilizada uma fonte radioativa de ${ }^{241} \mathrm{Am}$, um detector $2 \times 2$ " de NaI (Tl), ambos com colimadores de 2,0 mm, acoplado a uma eletrônica nuclear padrão. Na figura 2 é apresentado um espectro característico da fonte de ${ }^{241} \mathrm{Am}$, onde entre parênteses são apresentadas as probabilidades absolutas de transição. A resolução em energia do sistema foi de cerca de 5\%. As amostras, compostas por quantidades variáveis de parafina e água salgada com salinidade de 35,5 $\mathrm{kg} \cdot \mathrm{m}^{-3}$, foram acondicionadas em uma caixa de epóxi reforçado com fibra de carbono.

Figura 2. Espectro característico da fonte de ${ }^{241} \mathrm{Am}$. As duas energias maiores são raios gama e o restante são raios $\mathrm{X}$.

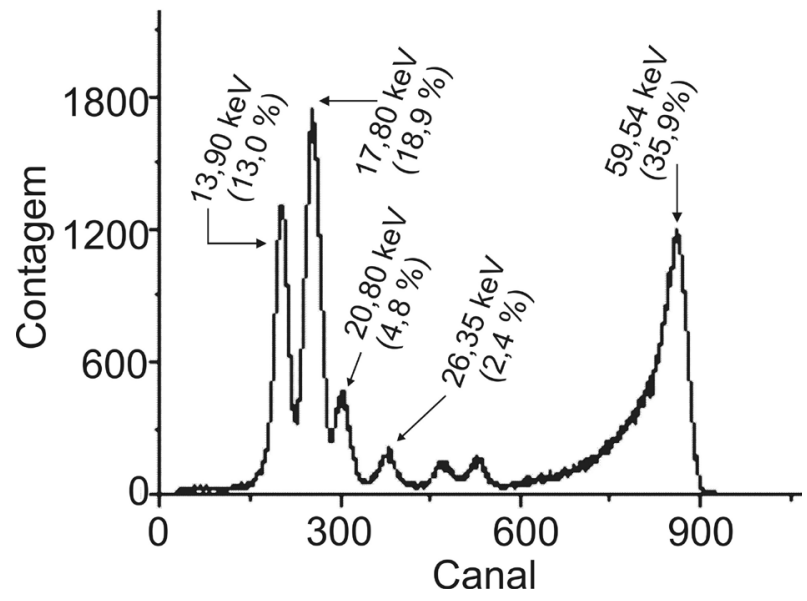

As medições foram realizadas com as linhas de 59,54 e 20,8 keV do ${ }^{241} \mathrm{Am}$. No intervalo de energia empregado a interação dos fótons com a matéria é principalmente devida ao efeito fotoelétrico e ao espalhamento Compton (o espalhamento elástico não é importante). $\mathrm{O}$ efeito fotoelétrico, que aumenta conforme diminui a energia, é muito mais sensível às variações da composição da amostra do que o espalhamento Compton, que aumenta com o aumento da energia, na faixa de energia utilizada.

Em contrapartida, como a energia diminui, a atenuação do feixe aumenta e a quantidade de contagem reduz a um nível que não permite uma boa estatística de contagem durante o tempo de medição. Então, como será mostrado, haverá níveis de energia específicos para se obter os melhores resultados.

Parafina foi utilizada para simular o petróleo, pois seu coeficiente de atenuação é semelhante ao dos tipos de petróleo usados atualmente. As medições de cada amostra foram repetidas 5 vezes. Para a linha de $20,8 \mathrm{keV}, 10 \%$ e $30 \%$ de água salgada com parafina foram testadas. Para a linha de $59,54 \mathrm{keV}$, as amostras foram preparadas com $20 \%, 70 \%$ e $90 \%$ de água salgada em volume para a mesma mistura.

O critério para o NMDC foi criado para garantir que um percentual definido da substância pode ser discriminado a partir do material puro. Este critério pode ser resumido da seguinte forma:

- Compara-se os valores de I / Io da mistura com I / Io da amostra pura, tendo como algarismos significativos até a posição correspondente a dois algarismos significativos do desvio-padrão;

- Compara-se a faixa de valores de I / Io da amostra pura e da mistura considerada, utilizando o intervalo (I/Io $\left.\pm 1,96 \sigma_{\mathrm{I} / \mathrm{o}}\right)$;

- Para considerar que a mistura é discriminada em relação à amostra pura, os dois intervalos de valores de I / Io precisam ser comparados, e deve haver uma diferença de duas unidades no último 
algarismo significativo.

Para testar este critério foram consideradas as estatísticas do desvio para a I e Io de: $0,3,0,5,1,2$, $3,5,10$ e $15 \%$. Onde:

- $0,3 \%$ corresponde a 100.000 contagens de I e Io.

- $0,5 \%$ corresponde a 40.000 contagens de I e Io.

- $1 \%$ corresponde a 10.000 contagens de I e Io.

- $2 \%$ corresponde a 2500 contagens para I e Io.

- 3\% corresponde a 1111 contagens para I e Io.

- $5 \%$ corresponde a 400 contagens de I e Io.

- $10 \%$ corresponde a 100 contagens para I e Io.

- $15 \%$ corresponde a 44 contagens de I e Io.

A etapa seguinte é comparar a relação I/Io da substância pura com a relação I/Io da mistura bifásica e verificar se existe diferença significativa, de acordo com o critério apresentado para NMDC.

\section{Resultados}

Como o epóxi utilizado é um material especial, foi necessária a obtenção da sua densidade experimentalmente, via método gravimétrico e o valor obtido foi $\rho_{\text {epóxi }}=(1,45 \pm 0,16) \cdot 10^{3} \mathrm{~kg} \cdot \mathrm{m}^{-3}$, determinando suas dimensões com um paquímetro e o volume imergindo em um béquer. Para determinar o coeficiente de atenuação linear, para a linha de $59,54 \mathrm{keV}$, dez placas de epóxi foram utilizadas, com espessura média de $2 \mathrm{~mm}$ cada.

Inicialmente foi utilizada uma placa, em seguida, duas, três e assim por diante até completar dez placas, com oito repetições em cada medição do feixe atenuado em relação ao índice inicial (I/ I0). Cada medida durou um minuto. Interpolando os valores da espessura para o $\ln (\mathrm{I} / \mathrm{I} 0)$ foi obtido o valor para cada grupo, $\mu=(49,51 \pm 0,11) \mathrm{m}^{-1}$.

Para efeito de comparação e validação, usando a fórmula molecular do epóxi, $\mathrm{C}_{39} \mathrm{H}_{82} \mathrm{O}_{10} \mathrm{Cl}_{28}$ (EPOXI, 2010), foi determinado o coeficiente de atenuação de massa com o programa WinXcom, para a linha de energia de $59,54 \mathrm{keV}$. O produto do coeficiente de atenuação de massa com densidade do epóxi, resultou no coeficiente de atenuação linear $\mu_{\text {epóxi }}=(49,65 \pm 0,09) \mathrm{m}^{-1}$. Como houve boa concordância entre os valores teóricos e experimentais para o coeficiente de atenuação linear, o valor teórico foi utilizado para o cálculo dos coeficientes de atenuação das outras linhas de energia do ${ }^{241} \mathrm{Am}$. Os resultados são apresentados na tabela 1 .

Tabela 1. Coeficientes de atenuação de massa e linear.

\begin{tabular}{lccccc}
\hline \multirow{2}{*}{$\begin{array}{l}\text { Energia } \\
(\mathbf{k e V})\end{array}$} & \multicolumn{2}{c}{ EPÓXI } & & \multicolumn{2}{c}{ PARAFINA } \\
\cline { 2 - 3 } \cline { 5 - 6 } & $\boldsymbol{\mu}_{\text {mass }}$ & $\boldsymbol{\mu}_{\text {linear }}$ & $\boldsymbol{\mu}_{\text {mass }}$ & $\boldsymbol{\mu}_{\text {linear }}$ \\
& $\left(10^{-1} \mathrm{~m}^{2} \cdot \mathrm{kg}^{-1}\right)$ & $\left(\mathrm{m}^{-1}\right)$ & $\left(10^{-1} \mathrm{~m}^{2} \cdot \mathrm{kg}^{-1}\right)$ & $\left(\mathrm{m}^{-1}\right)$ \\
\hline 59,54 & 0,3420 & 49,65 & 0,2000 & 16,72 \\
26,35 & 2,1800 & 316,50 & 0,3050 & 25,50 \\
20,80 & 4,2300 & 614,14 & 0,4060 & 33,95 \\
17,80 & 6,6000 & 958,23 & 0,5240 & 43,82 \\
13,90 & 13,500 & 1960,00 & 0,8780 & 73,42 \\
\hline
\end{tabular}

Da mesma forma, foi determinada a densidade e o coeficiente de atenuação linear da parafina, das quais cinco peças de parafina, de espessuras variadas foram usadas. O valor obtido para a densidade da parafina foi $\rho=(0,8362 \pm 0,0073) \cdot 10^{3}$ kg. $\mathrm{m}^{-3}$, o coeficiente de atenuação linear obtido experimentalmente foi $\mu=(17,70 \pm 0,09) \mathrm{m}^{-1}$ para a linha de 59,54 keV. Os valores dos coeficientes de atenuação obtidos com o programa WinXcom são mostrados na tabela 1 . Como pode ser visto na Tabela 2, o valor do coeficiente de atenuação dos quatro tipos de petróleo (CENTRAL EUROPEAN PETROLEUM, 2008) são semelhantes à da parafina, portanto, seu valor foi utilizado no experimento. 
Tabela 2. Coeficiente de atenuação de massa $\left(10^{-1}\right.$ $\mathrm{m}^{2} . \mathrm{kg}^{1}$ ) dos elementos usados no trabalho.

\begin{tabular}{|c|c|c|c|c|c|c|}
\hline$\gamma$ & $\begin{array}{c}\text { Energia } \\
(\mathrm{keV})\end{array}$ & 59,54 & 26,35 & 20,80 & 17,80 & 13,90 \\
\hline \multicolumn{7}{|l|}{ Material } \\
\hline \multicolumn{2}{|c|}{ Petr. Genérico } & 0,196 & 0,322 & 0,448 & 0,594 & 1,030 \\
\hline \multicolumn{2}{|c|}{ Petróleo A } & 0,199 & 0,396 & 0,409 & 0,530 & 0,893 \\
\hline \multicolumn{2}{|c|}{ Petróleo B } & 0,201 & 0,306 & 0,407 & 0,525 & 0,879 \\
\hline \multicolumn{2}{|c|}{ Petróleo Bell } & 0,178 & 0,295 & 0,412 & 0,550 & 0,963 \\
\hline \multicolumn{2}{|c|}{ Gás } & 0,212 & 0,310 & 0,402 & 0,509 & 0,830 \\
\hline \multicolumn{2}{|c|}{ Acrílico } & 0,193 & 0,357 & 0,529 & 0,732 & 1,340 \\
\hline \multicolumn{2}{|c|}{ Epóxi } & 0,342 & 2,180 & 4,230 & 6,600 & 13,50 \\
\hline \multicolumn{2}{|c|}{ Água } & 0,207 & 0,462 & 0,741 & 1,070 & 2,060 \\
\hline \multirow{7}{*}{$\begin{array}{c}\text { Água } \\
\text { salgada } \\
\left(\mathrm{kg} \cdot \mathrm{m}^{-3}\right)\end{array}$} & 35,5 & 0,212 & 0,532 & 0,884 & 1,300 & 2,530 \\
\hline & 50 & 0,214 & 0,560 & 0,940 & 1,390 & 2,720 \\
\hline & 100 & 0,221 & 0,648 & 1,120 & 1,670 & 3,310 \\
\hline & 150 & 0,227 & 0,729 & 1,290 & 1,940 & 3,850 \\
\hline & 200 & 0,232 & 0,804 & 1,440 & 2,180 & 4,350 \\
\hline & 250 & 0,237 & 0,872 & 1,580 & 2,400 & 4,810 \\
\hline & 300 & 0,242 & 0,935 & 1,700 & 2,600 & 5,230 \\
\hline \multicolumn{2}{|c|}{$\mathrm{Ar}$} & 0,187 & 0,424 & 0,681 & 0,984 & 1,890 \\
\hline
\end{tabular}

Os ajustes foram feitos para a relação I/Io versus a fração de água salgada em petróleo, para as cinco linhas de energia do ${ }^{241} \mathrm{Am}$, com várias salinidades e usando a caixa de acrílico. A Figura 3 mostra o gráfico da previsão teórica para a linha de $26,35 \mathrm{keV}$ para sete salinidades. Observou-se que as linhas de 26,35 e 20,80 keV foram as que apresentaram melhor discriminação, porque as linhas de 13,90 e 17,80 keV foram muito atenuadas, enquanto a linha de $59,54 \mathrm{keV}$ foi muito pouco atenuada.

As tabelas 3, 4 e 5 apresentam os dados referentes ao cálculo teórico do NMDC por três diferentes misturas bifásicas, com as cinco linhas de energia do ${ }^{241} \mathrm{Am}$. Para o caso do petróleo, apenas a tabela com petróleo A foi apresentada, visto todos serem semelhantes. Para a caixa de epóxi, apenas as três linhas de energia mais elevadas foram apresentadas.

Para a validação das previsões teóricas, as medidas foram realizadas com as linhas de $20,8 \mathrm{keV}$ e 59,54 $\mathrm{keV}$, com água salgada em parafina, usando a caixa de epóxi. Os resultados são mostrados na tabela 6. Como a fração da amostra era conhecida, uma comparação entre os percentuais previamente conhecidos e aqueles medidos por transmissão gama foi feita. Os cálculos foram feitos usando a equação 1 e o ajuste dos resultados está apresentado na equação (2),
$A m=5,2+0,90 * \mathrm{Ac}$,

Onde Am representa a fração percentual de amostra medida e Ac representa a fração percentual da amostra conhecida. O resultado mostra um bom ajuste e valida o modelo teórico, pois $\mathrm{R}^{2}=0,978$.

Figura 3. NMDC de água salgada em petróleo A para a energia de $26.35 \mathrm{keV}$. Salinidade apresentada em $\mathrm{kg} \cdot \mathrm{m}^{-3}$.

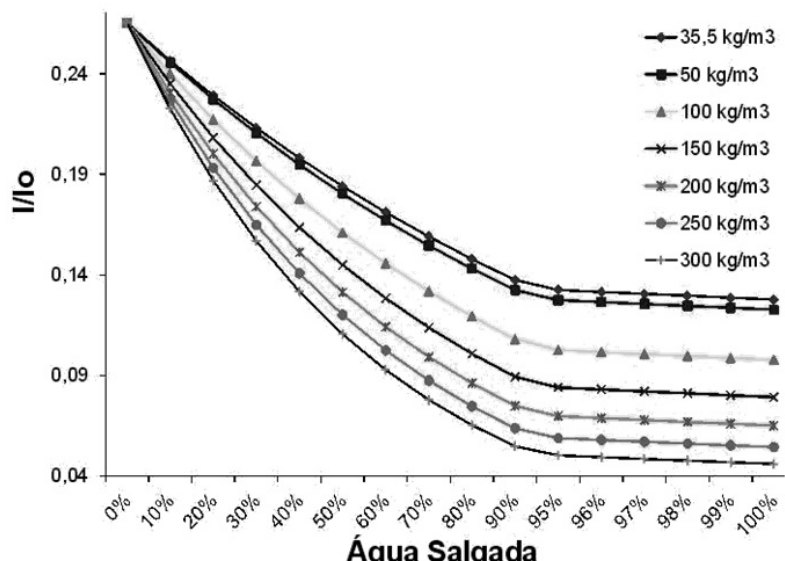

Tabela 3. NMDC da água salgada $\left(35.5 \mathrm{~kg} \cdot \mathrm{m}^{-3}\right)$ em gás usando caixa de acrílico.

\begin{tabular}{lccc}
\hline $\begin{array}{l}\text { Energia } \\
(\mathbf{k e V})\end{array}$ & $\begin{array}{c}\text { Estatística } \\
\text { de I e Io }(\%)\end{array}$ & $\begin{array}{c}\text { NMDC } \\
(\mathbf{\%})\end{array}$ & $\begin{array}{c}\text { I/Io de } \\
\text { NMDC }\left(.10^{-1}\right)\end{array}$ \\
\hline 13,9 & 15 & 5 & 1,22 \\
& 5 & 3 & 1,36 \\
& 2 & 1 & 1,52 \\
\hline 17,8 & 15 & 20 & 2,01 \\
& 10 & 10 & 2,62 \\
& 3 & 5 & 2,99 \\
& 2 & 1 & 3,32 \\
\hline 20,8 & 15 & 30 & 2,62 \\
& 10 & 20 & 3,11 \\
& 5 & 10 & 3,69 \\
& 2 & 5 & 4,02 \\
& 0.5 & 1 & 4,31 \\
\hline 26,35 & 15 & 95 & 2,23 \\
& 10 & 40 & 3,74 \\
& 5 & 20 & 4,51 \\
& 2 & 10 & 4,95 \\
& 0.5 & 2 & 5,34 \\
\hline 59,54 & 5 & 90 & 5,40 \\
& 3 & 50 & 5,79 \\
& 2 & 40 & 6,13 \\
& 0.5 & 10 & 6,61 \\
\hline & & &
\end{tabular}


Tabela 4. NMDC do gás em petróleo A.

\begin{tabular}{|c|c|c|c|c|c|}
\hline E (keV) & $\begin{array}{c}\text { Estatistica } \\
\text { I e Io(\%) }\end{array}$ & $\begin{array}{l}\text { NMDC } \\
(\%)^{1}\end{array}$ & $\begin{array}{c}\text { I/Io } \\
\text { de } \text { NMDC }^{1}\end{array}$ & $\begin{array}{c}\text { NMDC } \\
(\%)^{2}\end{array}$ & $\begin{array}{c}\text { I/Io de } \\
\text { NMDC }^{2}\end{array}$ \\
\hline \multirow[t]{6}{*}{13.9} & 15 & 20 & $9,05 \cdot 10^{-2}$ & - & - \\
\hline & 10 & 10 & $8,43 \cdot 10^{-2}$ & - & \\
\hline & 5 & 5 & $8,07 \cdot 10^{-2}$ & - & - \\
\hline & 3 & 3 & $8,02 \cdot 10^{-2}$ & - & - \\
\hline & 2 & 2 & $7,96 \cdot 10^{-2}$ & - & - \\
\hline & 0.5 & 1 & $7,90 \cdot 10^{-2}$ & - & - \\
\hline \multirow[t]{7}{*}{17.8} & 15 & 80 & $2,29 \cdot 10^{-2}$ & - & - \\
\hline & 10 & 50 & $2,04 \cdot 10^{-2}$ & - & - \\
\hline & 5 & 40 & $1,96 \cdot 10^{-2}$ & - & - \\
\hline & 3 & 20 & $1,81 \cdot 10^{-2}$ & - & - \\
\hline & 2 & 10 & $1,73 \cdot 10^{-2}$ & - & - \\
\hline & 0.5 & 4 & $1,69 \cdot 10^{-2}$ & - & - \\
\hline & 0.1 & 2 & $1,68 \cdot 10^{-2}$ & - & - \\
\hline \multirow[t]{7}{*}{20.8} & 15 & 90 & $2,80 \cdot 10^{-2}$ & - & - \\
\hline & 10 & 80 & $2,71.10^{-2}$ & 90 & $3,99 \cdot 10^{-1}$ \\
\hline & 5 & 50 & $2,48.10^{-1}$ & 50 & $3,54 \cdot 10^{-1}$ \\
\hline & 3 & 20 & $2,27.10^{-1}$ & 30 & $3,34 \cdot 10^{-1}$ \\
\hline & 2 & 10 & $2,21.10^{-1}$ & 20 & $3,24 \cdot 10^{-1}$ \\
\hline & 0.5 & - & - & 5 & $3,10.10^{-1}$ \\
\hline & 0.1 & 1 & $2,15 \cdot 10^{-1}$ & - & - \\
\hline \multirow[t]{6}{*}{26.35} & 5 & 60 & $3,01.10^{-1}$ & 80 & $4,47 \cdot 10^{-1}$ \\
\hline & 3 & 50 & $2,94 \cdot 10^{-1}$ & 60 & $4,29.10^{-1}$ \\
\hline & 2 & 20 & $2,77.10^{-1}$ & 50 & $4,20.10^{-1}$ \\
\hline & 1 & 10 & $2,71.10^{-1}$ & - & - \\
\hline & 0.5 & - & - & 10 & $3,86.10^{-1}$ \\
\hline & 0.1 & 1 & $2,66.10^{-1}$ & - & - \\
\hline \multirow[t]{5}{*}{59.54} & 3 & 90 & $3,69 \cdot 10^{-1}$ & - & - \\
\hline & 2 & 40 & $3,48 \cdot 10^{-1}$ & 70 & $5,14 \cdot 10^{-1}$ \\
\hline & 1 & 20 & $3,39 \cdot 10^{-1}$ & - & - \\
\hline & 0.5 & - & - & 20 & $4,84 \cdot 10^{-1}$ \\
\hline & 0.1 & 1 & $3,32 \cdot 10^{-1}$ & - & - \\
\hline
\end{tabular}

${ }^{1}$ Para acrílico; ${ }^{2}$ Para epóxi 
Tabela 5. NMDC de água salgada em petróleo A.

\begin{tabular}{|c|c|c|c|c|c|}
\hline E (keV) & $\begin{array}{c}\text { Estatistica } \\
\text { I e Io(\%) }\end{array}$ & $\begin{array}{c}\text { NMDC } \\
(\%)^{1} \\
\end{array}$ & $\begin{array}{c}\text { I/Io } \\
\text { de } \text { NMDC }^{1} \\
\end{array}$ & $\begin{array}{c}\text { NMDC } \\
(\%)^{2} \\
\end{array}$ & $\begin{array}{c}\text { I/Io de } \\
\text { NMDC }^{2}\end{array}$ \\
\hline \multirow[t]{2}{*}{13.9} & 15 & 2 & $7,15 \cdot 10^{-2}$ & - & - \\
\hline & 3 & 1 & $7,49 \cdot 10^{-2}$ & - & - \\
\hline \multirow[t]{4}{*}{17.8} & 15 & 20 & $1,07 \cdot 10^{-1}$ & - & - \\
\hline & 10 & 10 & $1,33 \cdot 10^{-1}$ & - & - \\
\hline & 3 & 2 & $1,59 \cdot 10^{-1}$ & - & - \\
\hline & 0.5 & 1 & $1,63 \cdot 10^{-1}$ & - & - \\
\hline \multirow[t]{5}{*}{20.8} & 15 & 20 & $1,61.10^{-1}$ & 20 & 0,230 \\
\hline & 5 & 10 & $1,86.10^{-1}$ & 10 & 0,265 \\
\hline & 3 & 3 & $2,05 \cdot 10^{-1}$ & - & - \\
\hline & 2 & - & - & 3 & 0,293 \\
\hline & 0.5 & 2 & $2,08 \cdot 10^{-1}$ & 1 & 0,301 \\
\hline \multirow[t]{6}{*}{26.35} & 15 & 40 & $1,98 \cdot 10^{-1}$ & 50 & 0,263 \\
\hline & 10 & 30 & $2,13 \cdot 10^{-1}$ & 40 & 0,283 \\
\hline & 5 & - & - & 20 & 0,327 \\
\hline & 3 & 20 & $2,29 \cdot 10^{-1}$ & - & - \\
\hline & 2 & 10 & $2,47.10^{-1}$ & - & - \\
\hline & 0.5 & 2 & $2,61.10^{-1}$ & 3 & 0,370 \\
\hline \multirow[t]{5}{*}{59.54} & 5 & 95 & $2,92 \cdot 10^{-1}$ & - & - \\
\hline & 3 & 80 & $2,98 \cdot 10^{-1}$ & 90 & 0,420 \\
\hline & 2 & 40 & $3,14 \cdot 10^{-1}$ & 70 & 0,431 \\
\hline & 0.5 & 20 & $3,23.10^{-1}$ & 20 & 0,460 \\
\hline & 0.1 & 2 & $3,30 \cdot 10^{-1}$ & - & - \\
\hline
\end{tabular}

${ }^{1}$ Para acrílico; ${ }^{2}$ Para epóxi

Tabela 6. NMDC de água salgada em parafina.

\begin{tabular}{ccccc}
\hline $\mathbf{E}$ & $\begin{array}{c}\text { Estatistica } \\
\text { I e Io }(\mathbf{\%})\end{array}$ & $\begin{array}{c}\text { NMDC } \\
(\mathbf{\%})\end{array}$ & $\begin{array}{c}\text { NMDC } \\
\text { NMDC (.10-1) }\end{array}$ & Tempo (s) \\
\hline 20,8 & 10 & 30 & 4,76 & 18 \\
& 5 & 10 & 4,88 & 7 \\
59,54 & 3 & 90 & 4,719 & 3 \\
& 3 & 70 & 4,997 & 6 \\
& 2 & 20 & 5,040 & 24 \\
\hline
\end{tabular}




\section{Conclusões}

De acordo com as tabelas 3 a 5 de NMDC, é possível discriminar uma substância em um líquido bifásico com um nível mínimo desejável de discriminação, sem a necessidade do número de contagens elevadas. Também foi observado que as melhores energias para discriminar todas as misturas são as intermediárias de 17,8, 20,8 e $26,35 \mathrm{keV}$, visto que a linha de $13,9 \mathrm{keV}$ é muito atenuada, enquanto a de 59,54 keV não é atenuada o suficiente, o que implica em I e I0 apresentarem valores muito próximos.

No entanto, é de extrema importância incluir neste critério o uso da linha de $59,54 \mathrm{keV}$, visto que se a espessura da amostra for elevada, as energias inferiores podem ser muito atenuadas. Um bom resultado foi obtido a partir da comparação dos dados experimentais com os cálculos de modelagem matemática. A modelagem matemática é aplicável para orientar o planejamento de experimentos para determinar as frações de uma substância em um líquido bifásico envolvendo petróleo, gás natural ou de água salgada. Foi obtida uma boa correlação entre os valores conhecidos da mistura bifásica e aqueles medidos por transmissão, conforme apresentado na equação 2 , comprovando que a metodologia apresenta boa aplicabilidade.

\section{Referências}

CENTRAL EUROPEAN PETROLEUM. Descrição da composição media do petróleo. Disponível em: $<\mathrm{http}$ :// www.cepetro.com/> Acesso em: 25 Fev. 2008.

EPOXI. Silaex Química Ltda. Chemical formula of the epoxy. Disponível em: $<\mathrm{http}$ ://www.silaex.com.br/epoxi. htm>. Acesso em: 20 Jan. 2010.

GERWARD, N.; GUILBERT, B.; JENSEN, K. B.; LEVRING, H. X-ray absorption in matter: reengineering XCOM. Radiation Physics and Chemistry, Oxford, v. 60, p. 23, 2001.

JOHANSEN, G. A.; JACKSON G. A. Salinity independent measurement of gas volume fraction in oil/ gas/water pipe flows. Applied Radiation and Isotopes, Oxford, v. 53, n. 4-5, p. 595-601, 2000.
PUTZ, A. G.; MORINEAU, Y. M.; BEGANI, R.; AQUITAINE, E.; Gamma ray absorption measurements, laboratory experiments in bottomhole conditions In: SOCIETY PETROLEUM ENGINEERS ANNUAL TECHNICAL CONFERENCE AND EXHIBITION, 1993, Texas. Proceedings... Texas: SPE, 1993.

ROACH, G. J.; WATT, J. S.; ZAATAWNY, H. W.; HARTLEY, P. E.; ELLIS, W. K. Multiphase flow meter for oil, water and gas in pipelines based on gamma-ray transmission techniques. Nuclear Geophysics, Oxford, v. 8, n. 3, p. 225, 1994.

SCHEERS, A. M.; SLIJKERMAN, W. F. J. Multiphase flow measurement using multiple energy gamma ray absorption (MEGRA) composition measurement. In: SOCIETY PETROLEUM ENGINEERS ANNUAL TECHNICAL CONFERENCE AND EXHIBITION, 1996, Denver. Proceedings... Denver: SPE, 1996.

STRAPASSON, A. B., NETO A. S., SOLETTO, A. T., BRIGHENTI, C. R. F. Geologia do petróleo. Disponível em: $<$ http://geocities.yahoo.com.br/geologiadopetroleo/ bacias.htm $>$. Acesso em: 12 jun. 2010.

TJUGUM, S. A.; JOHANSEN, G. A.; HOSTAD, M. B. The use of gamma radiation in fluid flow measurements. Radiation Physics and Chemistry, Oxford, v. 61, p. 797, 2001.

Recebido em 14 Janeiro 2011- Received on January 14, 2011.

Aceito em 14 Julho, 2011 - Accepted on July 14, 2011. 